\title{
Cognitive Effects of Subthalamic Nucleus Stimulation in Parkinson's Disease: A Controlled Study
}

\author{
Carmen Sáez-Zea ${ }^{a, c}$ Francisco Escamilla-Sevilla ${ }^{a}$ Majed J. Katati ${ }^{b}$ \\ Adolfo Mínguez-Castellanos ${ }^{a}$ \\ Departments of a Neurology and ${ }^{\mathrm{b}}$ Neurosurgery, Movement Disorders Unit, Virgen de las Nieves University \\ Hospital, Granada, and 'Department of Psychology, Area of Psychobiology, Faculty of Humanities and Education, \\ University of Jaén, Jaén, Spain
}

\section{Key Words}

Cognition - Deep brain stimulation - Neuropsychological testing $\cdot$ Parkinson's disease $\cdot$ Subthalamic nucleus $\cdot$ Verbal fluency

\begin{abstract}
Background: Subthalamic nucleus deep brain stimulation (STN-DBS) improves motor function in selected patients with Parkinson's disease (PD) but can be associated with variable changes in cognitive functions. Methods: We studied 21 patients selected for STN-DBS and compared 6-month clinical and neuropsychological outcomes between those who underwent surgery $(n=9)$ and those who voluntarily refused it $(n=12)$. Results: Motor and quality of life outcomes were markedly superior in the STN-DBS group versus controls. A wide neuropsychological battery was administered, and the whole sample showed a statistically significant worsening in phonemic verbal fluency, time to perform the Trail Making Test part B, Digit Symbol score of WAIS-III and color-naming score of the Stroop Test. In comparison to controls, a trend to a slightly worse deterioration in phonemic verbal fluency was observed in the STN-DBS patients and was significantly correlated with reductions in the L-dopa-equivalent daily dose $(r=0.850, p=0.007)$. Conclusion:
\end{abstract}

Our study confirms the safety of STN-DBS from a cognitive standpoint; a reduction in verbal fluency at 6 months after surgery can also be related to PD progression and medication reduction.

Copyright $\odot 2012$ S. Karger AG, Basel

\section{Introduction}

Deep brain stimulation of the subthalamic nucleus (STN-DBS) is an effective surgical procedure to improve motor symptoms and quality of life in selected patients with Parkinson's disease (PD) [1-3]. Numerous studies have assessed the short- and long-term neuropsychological changes after STN-DBS; in the absence of complications, it appears to be a safe procedure from a cognitive standpoint, although changes have been reported in executive functions, especially in verbal fluency tasks [4]. However, the progression of PD is also accompanied by neuropsychological deterioration, and controlled trials are required to establish the contribution of STN-DBS and to clarify the role of other factors, including the intracranial surgery itself, electrical stimulation and medication changes. 
Table 1. Neuropsychological assessment

\begin{tabular}{ll}
\hline Cognitive domains & Tests \\
\hline Orientation & Personal, time, place (WMS-III-R) \\
\hline Attention & $\begin{array}{l}\text { Backwards digit span (WAIS-III) } \\
\text { Trail Making Test parts A and B } \\
\text { Stroop Test } \\
\text { The'A' Test }\end{array}$ \\
\hline Verbal memory & Digits (WAIS-III) \\
& Auditory-Verbal Learning Test \\
\hline Motor coordination & Rhythmic sequences \\
& Motor inhibition \\
& Luria's fist-edge-palm test \\
\hline Language & Boston Naming Test \\
& Semantic Verbal Fluency Test (animals) \\
& Phonemic Verbal Fluency Test (P/M/R) \\
\hline Praxias & Block Design (WAIS-III) \\
& Bimanual praxias \\
& Two-dimensional construction \\
Three-dimensional construction
\end{tabular}

WAIS-III = Wechsler Adult Intelligence Scale-3rd edition; WMS-iii-R = Wechsler Memory Scale-3rd edition-revised.

With this background, our objective was to study cognitive changes at 6 months after bilateral STN-DBS in comparison to controls with similar clinical characteristics and to identify possible associated factors.

\section{Methods}

\section{Study Design}

A prospective non-randomized controlled study was conducted in patients with advanced PD referred to our Movement Disorders Unit and considered candidates for STN-DBS, comparing 6-month outcomes between those who underwent surgery and those who voluntarily refused it. An analysis of the changes in body weight and other nutritional parameters was previously published [5]. In the present study, we analyzed neuropsychological outcomes in 21 patients who completed the 6-month followup and were extensively evaluated by the same investigator (C.S-Z.); 9 of these patients underwent bilateral STN-DBS and the remaining 12 refused surgery.
Patients

All of the 21 patients met the study selection criteria. Inclusion criteria were: diagnosis of idiopathic PD according to the CAPSIT-PD protocol [6]; >5-year history of PD; presence of motor complications causing functional disability and not controllable by pharmacologic treatment, and L-dopa test with improvement of $\geq 33 \%$ in part III of the Unified Parkinson's Disease Rating Scale (UPDRS-III). Exclusion criteria were: presence of another disease that has a poor medium-term survival/functional prognosis or increases the surgical risk; a marked functional disability or postural instability in 'on' state; cognitive impairment; severe active psychiatric disorder, and magnetic resonance imaging showing severe brain atrophy or anomalies that increase the surgical risk. The study was approved by the Ethics Review Committee of our institution, and written informed consent was obtained from all participants.

\section{Surgical Procedure}

The surgical technique has been described in detail elsewhere [7]. The localization of the electrodes was checked on the day of surgery by magnetic resonance imaging. Contact and stimulation parameters (frequency, pulse width and voltage) were selected to obtain the optimal clinical benefit in both 'off' and 'on' medication states, with subsequent adjustments as required. The 9 operated patients in this study underwent surgery without complications and completed 6 months of effective electrical stimulation. The medication dose was optimized in the weeks or months after surgery, with variable reductions according to the clinical symptoms.

\section{Clinical Assessments}

All 21 patients underwent an extensive neuropsychological assessment by the same researcher (C.S-Z.) at baseline, i.e. after their inclusion in the study (1-2 weeks before the surgery in operated patients), and again at 6 months. The battery of tests assessed various cognitive domains, including orientation, attention, memory, motor coordination, language, praxias and executive functions (table 1 lists the tests administered). Before the cognitive evaluation, we conducted a clinical interview to screen for behavioral or psychiatric disorders, using the Montgomery-Åsberg Depression Rating Scale to assess emotional aspects. All neuropsychological assessments were conducted in the optimal 'on' medication state and always in 'on' stimulation in the surgical patients at their 6-month assessment. The stimulation parameters and medication dose were adjusted during the first 3 months postsurgery and were stable for at least 3 months before the neuropsychological assessment.

Motor (UPDRS-III, Hoehn \& Yahr staging) and quality of life (PDQ-39 questionnaire) scale scores were also determined in all patients at baseline and at 6 months. These neurological assessments were conducted in 'off' and 'on' medication states, and always in 'on' stimulation in the surgical patients at their 6-month assessment. The L-dopa-equivalent daily dose (LEDD) was calculated according to commonly accepted equivalences of antiparkinsonian medications [3].

\section{Statistical Analysis}

Baseline characteristics were compared between groups by using the Mann-Whitney U test (quantitative variables) or the Fisher's exact test (categorical variables). Baseline and 6-month follow-up values of neuropsychological variables were compared by 
means of Wilcoxon's signed-rank test, and the magnitude of change was compared between the surgical and control group with the Mann-Whitney U test. Associations of neuropsychological changes with clinical changes and with predictive factors for neuropsychological outcome were analyzed by using paired Spearman correlation coefficients. All tests were non-parametric and two-tailed, and $\mathrm{p}<0.05$ was considered significant.

\section{Results}

Baseline characteristics of the STN-DBS and control groups are exhibited in table 2 . No statistically significant differences were found between them in demographic characteristics, educational level or main PD-related variables. However, although significance was not reached, the STN-DBS group had a lower mean age (54 vs. 62 years in controls), a higher proportion of males (89 vs. $58 \%$ in controls) and a higher educational level (67\% completed primary schooling vs. $42 \%$ in controls). Only small non-significant differences in clinical variables were observed between the groups (see table 2).

Clinical outcomes at 6 months were significantly better in the STN-DBS group than in the control group: in the operated patients, the median improvement in UPDRS score in 'off' was 41 versus $7 \%$ in controls, the median improvement in PDQ-39 questionnaire was $47 \%$ versus median worsening of $20 \%$ in controls, and the median reduction in LEDD was 19\% versus no reduction in controls. There were no differences between groups in mood outcomes.

The whole sample showed a statistically significant worsening in four variables out of all those considered in the neuropsychological tests administered: Phonemic Verbal Fluency Test (sum of words beginning with the letters ' $\mathrm{P}$ ', ' $\mathrm{M}$ ' and ' $\mathrm{R}$ ' recalled by the patient during $1 \mathrm{~min}$ for each letter), time to perform part B of the Trail Making Test, the Digit Symbol score of the Wechsler Adult Intelligence Scale-3rd edition (WAIS-III), and the colornaming score of the Stroop Test (table 3). There were no statistically significant intergroup differences in the magnitude of the worsening, although a trend was observed to a slightly worse deterioration in phonemic verbal fluency and color-naming score of the Stroop Test in the STN-DBS group, and in part B of the Trail Making Test in the controls (table 3; fig. 1).

The sole association found between neuropsychological and clinical changes was a correlation in the STNDBS group between a higher reduction in LEDD and a greater deterioration in the Phonemic Verbal Fluency Test $(\mathrm{r}=0.850, \mathrm{p}=0.007)$. The sole association between
Table 2. Baseline characteristics of the STN-DBS and control groups

\begin{tabular}{|c|c|c|c|}
\hline & $\begin{array}{l}\text { STN-DBS } \\
\text { group } \\
(\mathrm{n}=9)\end{array}$ & $\begin{array}{l}\text { Control } \\
\text { group } \\
(\mathrm{n}=12)\end{array}$ & $\mathrm{p}^{*}$ \\
\hline Age, years & $54(14)$ & $62(10)$ & NS \\
\hline \multicolumn{4}{|l|}{ Sex, n } \\
\hline Male & 8 & 7 & \multirow[t]{2}{*}{ NS } \\
\hline Female & 1 & 5 & \\
\hline \multicolumn{4}{|l|}{ Schooling, $\mathrm{n}$} \\
\hline$<$ Primary & 3 & 7 & \multirow[t]{3}{*}{ NS } \\
\hline Primary & 3 & 3 & \\
\hline Secondary & 3 & 2 & \\
\hline Duration of disease, years & $12(2)$ & $15(6)$ & NS \\
\hline \multicolumn{4}{|l|}{$\mathrm{H} \& \mathrm{Y}$ stage in 'off, $\mathrm{n}$} \\
\hline 3 & 2 & 5 & \multirow[t]{2}{*}{ NS } \\
\hline$>3$ & 7 & 7 & \\
\hline UPDRS-III in 'off & $43(15)$ & $49(11)$ & NS \\
\hline LEDD, mg & $1,529(640)$ & $1,359(340)$ & NS \\
\hline
\end{tabular}

$\mathrm{H} \& \mathrm{Y}=$ Hoehn \& Yahr staging (1-5 from lesser to greater severity); UPDRS-III = part III of the Unified Parkinson's Disease Rating Scale (0-108, with higher levels indicating greater severity); LEDD = L-dopa-equivalent daily dose (see text). Values are means (SD in parentheses) for quantitative variables, and absolute frequencies for categorical variables.

${ }^{*}$ p value: comparison between STN-DBS and control groups (Mann-Whitney U test for quantitative variables and Fisher's exact test for categorical variables); NS = not significant $(\geq 0.05)$.

baseline demographic or clinical characteristics (in table 2) and neuropsychological outcomes was a correlation between higher age and a worse deterioration in the time taken to complete part B of the Trail Making Test $(\mathrm{r}=$ $0.775, \mathrm{p}=0.003)$.

\section{Discussion}

Our study of outcomes at 6 months post-surgery confirms that STN-DBS is a safe procedure from a cognitive standpoint. Small changes in some tests of executive functions did not significantly differ from those observed in control patients and can therefore be largely attributed to the progression of PD. At any rate, these changes did not compromise the important gain in quality of life by the patients who received the surgery.

Although not statistically significant, a slightly greater deterioration in phonemic verbal fluency was observed in the STN-DBS patients than in the controls at 6 months, 
Fig. 1. Box-plots showing the changes in the Phonemic Verbal Fluency Test (PhVF; a) and in the time to perform the Trail Making Test part B (TMT-B; b) at the 6-month follow-up. Negative values denote reduction (worsening in Phonemic Verbal Fluency) and positive values increment (worsening in time to complete the Trail Making Test part B).
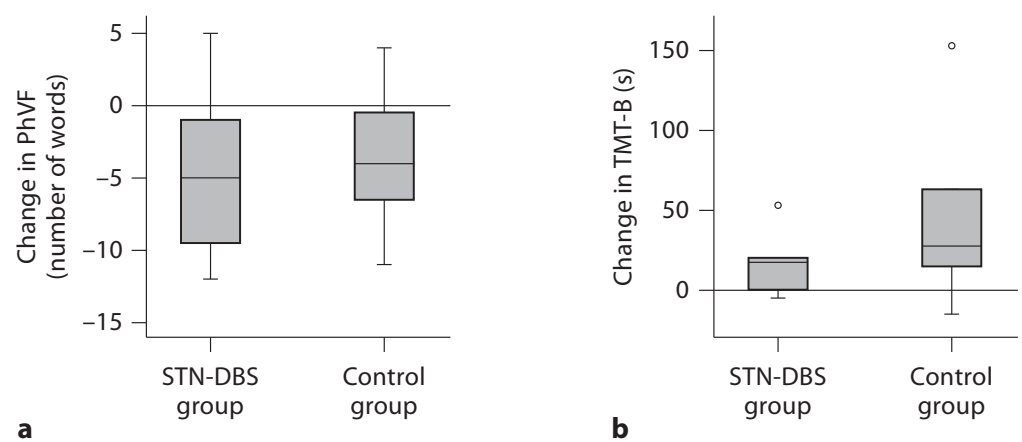

Table 3. Neuropsychological tests with statistically significant change at 6-month follow-up in the whole sample

\begin{tabular}{llllll}
$\begin{array}{l}\text { Baseline } \\
(\mathrm{n}=21)\end{array}$ & $\begin{array}{l}6 \text { months } \\
(\mathrm{n}=21)\end{array}$ & Change & $\mathrm{p}^{*}$ & $\mathrm{p}^{\dagger}$ \\
\cline { 3 - 5 } & $\begin{array}{l}\text { STN-DBS group } \\
(\mathrm{n}=9)\end{array}$ & $\begin{array}{l}\text { control group } \\
(\mathrm{n}=12)\end{array}$ & $\begin{array}{l}\text { total } \\
(\mathrm{n}=21)\end{array}$
\end{tabular}

Phonemic Verbal Fluency Test

(number of words)

Trail Making Test part B (s)

Digit Symbol

Stroop Test: color naming

$\begin{array}{ccc}35.95(15.35) & 31.89(14.73) & -4.75(5.82) \\ 107(36) & 151(49) & +17.17(20.45) \\ 19.73(9.66) & 16.22(10.60) & -2.80(1.79) \\ 38.53(9.91) & 34.63(8.09) & -9.33(5.60)\end{array}$

$$
\begin{gathered}
-3.36(4.59) \\
+45.17(58.50) \\
-2.78(3.70)
\end{gathered}
$$$$
-5.14(8.36)
$$

$$
\begin{gathered}
-3.95(5.04) \\
+31.17(44.27) \\
-2.79(3.07)
\end{gathered}
$$

-5.69 (6.69)
0.007 NS

0.010 NS

0.013 NS

0.016 NS

Values are means (SD). Performance of Trail Making Test part B is related to the educational level and could only be achieved by 12 patients (6 in each group). Scores for Digit Symbol and Stroop Test were adjusted by age. 'Change' refers to the mean (SD) of the individual changes in paired comparisons between the baseline and 6-month evaluations; plus sign denotes increment (worsening in Trail Making Test part B) and minus sign reduction (worsening in Phonemic Verbal Fluency, Digit Symbol and Stroop Test).

* p value: paired comparisons between baseline and 6-month evaluations in the whole sample (Wilcoxon's signed rank test).

$\dagger p$ value: comparison of changes between STN-DBS and control groups (Mann-Whitney U test); NS = not significant ( $\geq 0.05$ ).

suggesting that this may be the most consistent neuropsychological effect related to the procedure, as previously reported $[2,8]$. A similar trend was observed in the colornaming score of the Stroop Test, which is also related to executive functioning. In contrast, the STN-DBS patients showed a lesser deterioration versus controls in the time taken to complete the part B of the Trail Making Test, but this non-significant difference may be explained by the lower mean age of the group, given that age was found to be the main predictive factor for worsening in this variable.

Limitations of our study were the small sample size, which may account for failures to detect statistically significant differences, the medium-term follow-up and the absence of randomization. However, patients were prospectively selected and operated patients did not significantly differ from those who refused surgery (controls).
Refusal of surgery is a complex decision by the patient and his/her relatives which was not specifically investigated, although we cannot rule out a role for the nonsignificantly higher age or lower educational level of this group. Undoubtedly, this issue warrants further research.

The best-quality evidence on neuropsychological changes after bilateral STN-DBS derives from two randomized controlled studies $[2,8]$. In both studies, surgical and control groups exhibited deterioration in verbal fluency and other variables related to executive functions at the 6-month follow-up, with the surgical groups showing a significantly greater worsening. Non-randomized controlled studies with a longer follow-up (1-3 years) also reported that verbal fluency is the main impaired neuropsychological variable after the procedure [9-12].

Phonemic verbal fluency is the most commonly affected type, although deterioration in semantic verbal flu- 
ency has also been variably reported $[8,9,12,13]$. The contributions of intracranial surgery, electrical stimulation or medication reduction to this selective impairment are under debate. A recent randomized study comparing 3-month outcomes between 101 patients undergoing bilateral STN-DBS and 35 patients receiving the same surgery but without stimulation showed a slight reduction in verbal fluency in both groups, with no significant difference between them [14]. Therefore, the electrical stimulation does not appear to play a major role in verbal fluency reduction, which may be attributable to the natural progression of $\mathrm{PD}$, the intracranial surgery and/or changes in medication.

The influence of medication changes warrants further attention, because the LEDD is reduced in most patients who undergo STN-DBS. In the present study, a significant correlation was observed between higher reduction in LEDD and greater deterioration in phonemic verbal fluency in the surgical group, whereas the medication dose was not changed in controls, suggesting that medication reduction may be implicated in the verbal fluency decline. The influence of this factor in the abovementioned randomized study by Okun et al. [14] cannot be ruled out, because the medication dose was reduced in both the stimulated group and non-stimulated group, although the reduction was greater in the former (492 vs. $131 \mathrm{mg} /$ day, respectively). Medication changes may also have exerted an influence on the neuropsychological outcomes of randomized controlled trials that compared globus pallidus interna DBS (Gpi-DBS) with STNDBS. Comparison of the effects of unilateral stimulation of Gpi versus STN (right or left depending on predominant motor symptoms) at 7 months showed that the phonemic verbal fluency deteriorated in the STN-DBS group alone, suggesting a target-related effect [15]; however, the medication management differed between the groups, with an increase of $\sim 100 \mathrm{mg} /$ day after Gpi-DBS. A larger randomized study comparing outcomes of bilateral Gpi-DBS and STN-DBS at 2 years found a significantly greater deterioration in the processing speed index of the WAIS-III in the STN-DBS group, whereas reductions in phonemic and semantic verbal fluency were similar between groups [16]; the LEDD was reduced in both groups, although this reduction was greater in the STN-DBS patients (408 vs. $243 \mathrm{mg} /$ day in GpiDBS group). Taken together, these results suggest that further research is warranted to determine the effects of medication reduction on neuropsychological changes after STN-DBS.

Cognitive Effects of Subthalamic Stimulation
The pathophysiological mechanisms underlying STN-DBS-related impairment in verbal fluency or executive functions are not precisely known. The STN forms part of a broadly distributed neural network that encompasses motor, associative and limbic circuits. Positron emission tomography (PET) studies of glucose metabolism and regional cerebral blood flow after STNDBS have shown a reversal of the distinctive PD-related pattern of increased activity in the basal ganglia and relatively reduced activity in various areas of the cerebral cortex [17]. However, reports on post-surgical changes in the associative and limbic cortical areas have been highly varied, with patients showing increased, unchanged or decreased activity. In relation to neuropsychological outcomes, a PET study of 9 STN-DBS patients at 6 months revealed decreased metabolic activity in the left dorsolateral prefrontal and Broca cortical areas in the 3 patients whose verbal fluency was impaired [18]. With regard to the acute effects of electrical stimulation, a PET study of regional cerebral blood flow in 7 patients showed greater activation of a left-sided frontotemporal network and the right orbitofrontal cortex during a verbal fluency task when stimulation was off and lesser activation when stimulation was on, suggesting that interference with basal ganglia-thalamocortical circuits may underlie the verbal fluency deterioration [19]. However, Campbell et al. [20] studied blood flow changes in resting state (instead of during a cognitive task) in 29 patients and found no statistically significant changes between off and on stimulation conditions, highlighting a marked interindividual variability. Interestingly, those patients who showed blood flow increase under stimulation in the dorsolateral prefrontal or anterior cingulated cortices also exhibited a worsening of working memory or response inhibition performance, respectively.

In summary, there is adequate evidence to affirm that implantation of electrodes in the STN and its electrical stimulation may induce functional changes in neural networks involved in cognition, although the interindividual variability is high. In the absence of complications, neuropsychological outcomes after surgery may variably depend on individual factors such as PD phenotype, localization of the electrode/stimulating contact, spread of electrical current and medication changes. Deterioration in verbal fluency accompanies the progression of PD, and the additional mean decrease observed after STN-DBS in large studies appears to be mainly related to intracranial surgery and/or decreased dopaminergic stimulation of frontal areas due to medication reduction.

Eur Neurol 2012;68:361-366 365 


\section{Acknowledgments}

The authors thank the Centro de Investigación Biomédica en Red sobre Enfermedades Neurodegenerativas (CIBERNED; Prof. J. López-Barneo) and Medtronic Ibérica SA for financial support to our research group.

\section{Disclosure Statement}

C. Sáez-Zea was supported by grants from the CIBERNED and FIBAO. F. Escamilla-Sevilla and A. Mínguez-Castellanos received honoraria for lectures and travel grants to attend scientific meetings from Boehringer-Ingelheim, Eisai, Glaxo-SmithKline, Italfarmaco, Lundbeck, Medtronic, MSD, Novartis, Abbott and UCB Pharma. M.J. Katati has no conflicts of interest to disclose.

\section{References}

1 Deuschl G, Schade-Brittinger C, Krack P, Volkmann J, Schäfer H, Bötzel K, Daniels C, Deutschländer A, Dillmann U, Eisner W, Gruber D, Hamel W, Herzog J, Hilker R, Klebe S, Kloss M, Koy J, Krause M, Kupsch A, Lorenz D, Lorenzl S, Mehdorn HM, Moringlane JR, Oertel W, Pinsker MO, Reichmann H, Reuss A, Schneider GH, Schnitzler A, Steude U, Sturm V, Timmermann L, Tronnier V, Trottenberg T, Wojtecki L, Wolf E, Poewe W, Voges J; German Parkinson Study Group, Neurostimulation Section: A randomized trial of deep-brain stimulation for Parkinson's disease. N Engl J Med 2006; 355:896-908.

-2 Weaver FM, Follett K, Stern M, Hur K, Harris C, Marks WJ Jr, Rothlind J, Sagher O, Reda D, Moy CS, Pahwa R, Burchiel K, Hogarth P, Lai EC, Duda JE, Holloway K, Samii A, Horn S, Bronstein J, Stoner G, Heemskerk J, Huang GD; CSP-468 Study Group: Bilateral deep brain stimulation vs. best medical therapy for patients with advanced Parkinson disease: a randomized controlled trial. JAMA 2009;301:63-73.

3 Williams A, Gill S, Varma T, Jenkinson C, Quinn N, Mitchell R, Scott R, Ives N, Rick C, Daniels J, Patel S, Wheatley K; PD SURG Collaborative Group: Deep brain stimulation plus best medical therapy versus best medical therapy alone for advanced Parkinson's disease (PD SURG trial): a randomised, open-label trial. Lancet Neurol 2010;9:581591.

$\checkmark 4$ Parsons TD, Rogers SA, Braaten AJ, Woods SP, Tröster AI: Cognitive sequelae of subthalamic nucleus deep brain stimulation in Parkinson's disease: a meta-analysis. Lancet Neurol 2006;5:578-588.

5 Escamilla-Sevilla F, Pérez-Navarro MJ, Muñoz-Pasadas M, Sáez-Zea C, Jouma-Katati M, Piédrola-Maroto G, Ramírez-Navarro A, Mínguez-Castellanos A: Change of the melanocortin system caused by bilateral subthalamic nucleus stimulation in Parkinson's disease. Acta Neurol Scand 2011;124:275281.

-6 Defer GL, Widner H, Marié RM, Rémy P, Levivier M: Core assessment program for surgical interventional therapies in Parkinson's disease (CAPSIT-PD). Mov Disord 1999; 14:572-584.
7 Mínguez-Castellanos A, Escamilla-Sevilla F, Katati MJ, Martin-Linares JM, Meersmans M, Ortega-Moreno A, Arjona V: Different patterns of medication change after subthalamic or pallidal stimulation for Parkinson's disease: target related effect or selection bias? J Neurol Neurosurg Psychiatry 2005;76:3439.

8 Witt K, Daniels C, Reiff J, Krack P, Volkmann J, Pinsker MO, Krause M, Tronnier V, Kloss M, Schnitzler A, Wojtecki L, Bötzel K, Danek A, Hilker R, Sturm V, Kupsch A, Karner E, Deuschl G: Neuropsychological and psychiatric changes after deep brain stimulation for Parkinson's disease: a randomised, multicentre study. Lancet Neurol 2008;7:605-614.

-9 Cilia R, Siri C, Marotta G, De Gaspari D, Landi A, Mariani CB, Benti R, Isaias IU, Vergani F, Pezzoli G, Antonini A: Brain networks underlining verbal fluency decline during STN-DBS in Parkinson's disease: an ECD-SPECT study. Parkinsonism Relat Disord 2007;13:290-294.

10 Zangaglia R, Pacchetti C, Pasotti C, Mancini F, Servello D, Sinforiani E, Cristina S, Sassi M, Nappi G: Deep brain stimulation and cognitive functions in Parkinson's disease: a three-year controlled study. Mov Disord 2009;24:1621-1628.

11 Castelli L, Rizzi L, Zibetti M, Angrisano S, Lanotte M, Lopiano L: Neuropsychological changes 1-year after subthalamic DBS in PD patients: a prospective controlled study. Par-
kinsonism Relat Disord 2010;16:115-118.

12 Williams AE, Arzola GM, Strutt AM, Simpson R, Jankovic J, York MK: Cognitive outcome and reliable change indices two years following bilateral subthalamic nucleus deep brain stimulation. Parkinsonism Relat Disord 2011;17:321-327.

13 Gironell A, Kulisevsky J, Rami L, Fortuny N, García-Sánchez C, Pascual-Sedano B: Effects of pallidotomy and bilateral subthalamic stimulation on cognitive function in Parkinson disease. A controlled comparative study. J Neurol 2003;250:917-923.
14 Okun MS, Gallo BV, Mandybur G, Jagid J, Foote KD, Revilla FJ, Alterman R, Jankovic J, Simpson R, Junn F, Verhagen L, Arle JE, Ford B, Goodman RR, Stewart RM, Horn S, Baltuch GH, Kopell BH, Marshall F, Peichel D, Pahwa R, Lyons KE, Tröster AI, Vitek JL, Tagliati M; SJM DBS Study Group: Subthalamic deep brain stimulation with a constant-current device in Parkinson's disease: an open-label randomised controlled trial. Lancet Neurol 2012;11:140-149.

15 Okun MS, Fernandez HH, Wu SS, KirschDarrow L, Bowers D, Bova F, Suelter M, Jacobson CE 4th, Wang X, Gordon CW Jr, Zeilman P, Romrell J, Martin P, Ward H, Rodriguez RL, Foote KD: Cognition and mood in Parkinson's disease in subthalamic nucleus versus globus pallidus interna deep brain stimulation: the COMPARE trial. Ann Neurol 2009;65:586-595.

16 Follett KA, Weaver FM, Stern M, Hur K, Harris CL, Luo P, Marks WJ Jr, Rothlind J, Sagher O, Moy C, Pahwa R, Burchiel K, Hogarth P, Lai EC, Duda JE, Holloway K, Samii A, Horn S, Bronstein JM, Stoner G, Starr PA, Simpson R, Baltuch G, De Salles A, Huang GD, Reda DJ; CSP-468 Study Group: Pallidal versus subthalamic deep-brain stimulation for Parkinson's disease. N Engl J Med 2010; 362:2077-2091.

17 Stoessl AJ, Martin WW, McKeown MJ, Sossi V: Advances in imaging in Parkinson's disease. Lancet Neurol 2011;10:987-1001.

18 Kalbe E, Voges J, Weber T, Haarer M, Baudrexel S, Klein JC, Kessler J, Sturm V, Heiss WD, Hilker R: Frontal FDG-PET activity correlates with cognitive outcome after STN-DBS in Parkinson disease. Neurology 2009;72:42-49.

19 Schroeder U, Kuehler A, Lange KW, Haslinger B, Tronnier VM, Krause M, Pfister R, Boecker H, Ceballos-Baumann AO: Subthalamic nucleus stimulation affects a frontotemporal network: a PET study. Ann Neurol 2003;54:445-450.

20 Campbell MC, Karimi M, Weaver PM, Wu J, Perantie DC, Golchin NA, Tabbal SD, Perlmutter JS, Hershey T: Neural correlates of STN DBS-induced cognitive variability in Parkinson disease. Neuropsychologia 2008; 46:3162-3169. 